\title{
Transient Remoulding of Nails as A Sequitur to Docetaxel
}

\author{
Dr. Sundeep Chowdhry ${ }^{1^{*}}$, Dr. Archana J Lokhande ${ }^{2}$, Dr. Paschal D’Souza ${ }^{3}$ \\ ${ }^{1 *}$ Assistant Professor, Dept of Dermatology, ESI PGMSR, Basaidarapur, New Delhi, India \\ ${ }^{2}$ Senior Resident, ESI PGMSR, Basaidarapur, New Delhi \\ ${ }^{3}$ Director Professor, Dept of Dermatology, ESI PGMSR, Basaidarapur, New Delhi, India \\ suncutis@gmail.com
}

*Corresponding Author: Dr. Paschal D'Souza, Director Professor, Department of Dermatology, ESI PGMSR, Basaidarapur, New Delhi, India.

\section{Abstract}

Taxanes are antimicrotubular anticancer drugs used for the treatment of various types of carcinomas including sarcomas. Docetaxel and Pacetaxel are drugs belonging to this category and are known to cause many hematological as well as cutaneous side effects.The nail changes caused by docetaxel are relatively benign in nature and proportional to the number of therapy cycles.

We report a 30 year old patient diagnosed with retroperitoneal sarcoma who developed severe nail changes on administration of chemotherapeutic agent docetaxel and these changes reversed to normal on withdrawal of the drug.

Keywords: Docetaxel; nail changes

\section{INTRODUCTION}

Chemotherapeutic agents are known to cause various cutaneous adverse effects, including hair and nail changes. Amongst these agents the taxanegroup of drugs are associated with adverse effects commonly involving nails.

\section{CASE Report}

A 30 year old male patient presented in the outpatient oncosurgery department with the complaint of back pain and palpable abdominal lump and was subsequently diagnosed as a case of retropreitoneal sarcoma in 2013 after due investigations.In the following one year period he underwent surgical resection of the tumor and was initiated on adjuvant radiation therapy.

On follow up visits in 2016 it was found that the tumor had relapsed along with evidence of systemic metastases. The patient was started on 6 cycles of first line chemotherapy drugs namely, ifosfamide and doxarubicin.

Despite this treatment the patient showed evidence of hepatic and retroperitoneal metastases on PET scan and thus was subsequently put on second line of chemotherapeutic drugs. Three cycles of injection gemcitabine $\left(1730 \mathrm{mg} / \mathrm{cm}^{2} /\right.$ cycle), docetaxel $(130 \mathrm{mg} /$ $\mathrm{cm}^{2} /$ cycle) and pegfilgramstin were administered intravenously. While still on this treatment he started experiencing multiple systemic side effects such as fever, weakness, altered blood cell counts (neutropenia) as well as associated superadded fungal infection (tineacruris and corporis), hair fall and nail changes involving all the finger nails. There was swelling of fingers with associated pain and discharge from nail folds (Fig1). This resulted in restricted day to day activities subsequent to excessive pain and impaired grip of fingers. On local examination there was transverse leuckonychia,serous discharge from lateral nail folds along with paronychia was present but nail bed purpura, subungual hematoma or hemorrhagic discharge was absent. The discharge on microscopic examination for $\mathrm{KOH}$ and gram stain was negative.

The patient came to dermatology OPD for these nail changes and tinea infection. He was given antifungal treatment (tablet terbinafine $250 \mathrm{mg}$ once a day, tab 


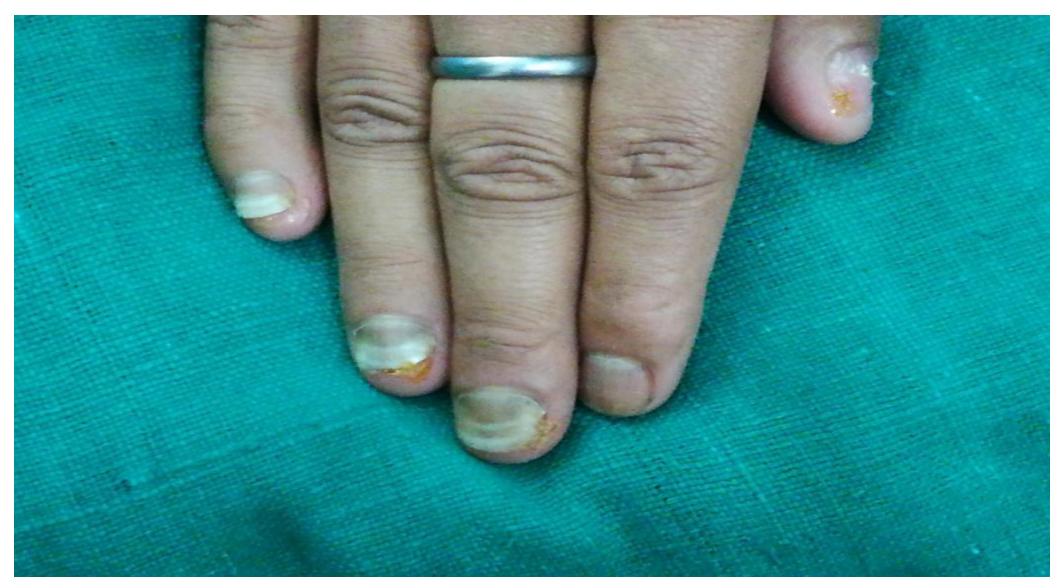

Figure 1. Paronychia with serous discharge, transverse leuckonychia

cetrizine $10 \mathrm{mg}$ once a day and sertaconazole cream twice a day for 3 weeks). After eliciting detailed history and relevant examination the patient was reassured for these nail changes and no treatment was prescribed.
On completion of the last therapy cycle the discharge stopped on its own in two weeks period and resulted in onycholysisi.e- separation of the nail plate from nail bed progressing from distal to proximal end (Fig 2) over next 6 weeks.

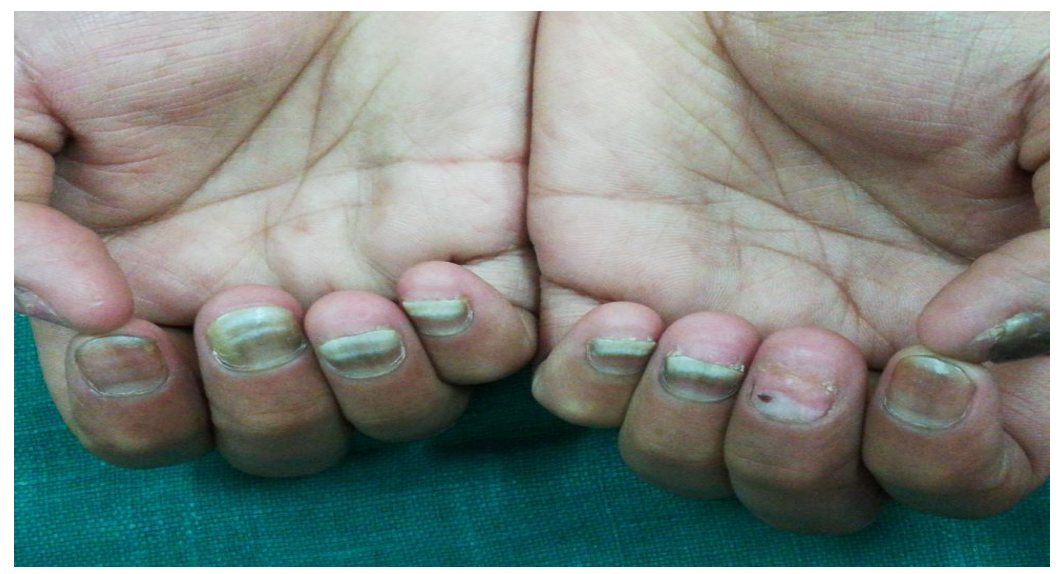

Figure 2. Onycholysis (after 2 months) post cessation of docetaxel

\section{DiscusSION}

Taxanes $^{1}$ (Docetaxel, Paclitaxel, Cabazitaxel ) are class of anti-cancer drugs, originally derived from natural sources and belongs to the class of mitotic inhibitor drugs. These drugs act by interfering with microtubules at the time of cell division process.

Docetaxel is a semi-synthetically derived drug which is widely used in management of variety of cancers of breast, ovary, lung, stomach, prostate, sarcomas, head and neck. ${ }^{2}$

Cutaneous side effects such as erythematous pruritic maculopapular rash, palmoplantarerythro-dysesthesia, xerosis and exfoliation are noted in $50-70 \%$ of patients who are on taxanes. ${ }^{3}$ The incidence of nail changes in patients on taxanes ranges from $0 \%$ to $44 \%$ as compared to other chemo-therapeputic agents. ${ }^{4}$

Nail abnormalities occurring during treatment with taxane are in most cases not serious in nature. The spectrum of nail involvement varies from nail pigmentation, transverse leukonychia, nail bed purpura, splinter hemorrhage, subungal hematoma, Beau's lines, acute paronychia, hemorrhagic onycholysis and subungual suppuration ${ }^{4,5}$, out of these the latter two are responsible for significant morbidity. The nail changes may be present in several or all nails, commonly involving finger nails than toe nails.

Possible accepted hypothesis for these nail changes are direct nail bed toxicity, thrombocytopenia and vascular abnormalities (due to inhibition of nail bed 
angiogenesis). These all are known taxans induced mediated adverse effects. ${ }^{4}$ Wasner $G$ et al has also proposed a neurogenic mechanism for docetaxel induced painful nail changes. ${ }^{6}$

In our patient transverse leuckonychia, subungual discharge, painful periungual swelling and onycholysis were seen only on initiation of second line chemotherapy. The nail changes reversed to normal after two months of stopping the second line of chemotherapeutic agents. These nail changes were not experienced by the patient while he was on first line of chemotherapy thus confirming their association with second line of chemotherapeutic agents. On searching the literature for the culprit agent amongst the two, namely docetaxel and gemcitabine, the latter was not found to be associated with these nail changes.

Docetaxel is frequently associated with these nail changes and its severity increases with number of chemotherapy cycles. Despite this, these changes are not critical in nature so as to necessitate stoppage of therapy. ${ }^{7}$ Treatment may be indicated if there is evidence of secondary bacterial or fungal infection due to onycholysis.

The split hand study conducted by Florian Scotté et al has recommended use of frozen gloves prior to, during and post docetaxel infusion to reduce the incidence and severity of skin and nail toxicity.

Minisini AM et $\mathrm{al}^{3}$ have reported spectrum of docetaxel induced nail changes in seven patients. In a case reported by Lehoczky et al, ${ }^{9}$ transverse leuchonychiya was reported with the use of another taxanei.e paclitaxel in patients with ovarian cancer.

\section{ConCLUSiON}

In our case nail changes appeared while the patient was on docetaxel chemotherapy and these changes resolved gradually over a period of few weeks after cessation of treatment. Though there are no preventive measures for avoiding the occurrence of these nail changes, the transient and benign nature of these seldom requires discontinuation of anticancer therapy.

\section{REFERENCES}

[1] Abal M, Andreu J M, BarasoainI.Taxanes: microtubule and centrosome targets, and cell cycle dependent mechanisms of action. Curr Cancer Drug Targets 2003 Jun;3(3):193-203

[2] Robert C, Sibaud V, Mateus C, Verschoore M, Charles C, Lanoy E, et al. Nail toxicities induced by systemic anticancer treatments. Lancet Oncol 2015;16 :e181-9.

[3] Pavithran K, Doval DC. Nail changes due to docetaxel. Br J Dermatol. 2002;146:709-10.

[4] Minisini AM, Tosti A, Sobrero AF, Mansutti M, Piraccini BM, Sacco C, et al. Taxane-induced nail changes: incidence, clinical presentation and outcome. Ann Oncol. 2003;14:333-7.

[5] Roh MR, Cho JY, Lew W. Docetaxel-Induced Onycholysis: The Role of Subungual Hemorrhage and Suppuration. Yonsei Medical Journal 2007;48(1):124-6.

[6] Wasner G, Hilpert F, Schattschneider J, Binder A, Pfisterer J, Baron R. Docetaxel-induced nail changes -a neurogenic mechanism: a case report. J Neurooncol 2002;58:167-74.

[7] Ho, Maria Y and John R Mackey."Presentation and Management of Docetaxel -Related Adverse Effects in Patients with Breast Cancer." Cancer Management and Research 2014;6: 253-9.

[8] Scotté. F, Tourani. J.M ,Banu.E , Peyromaure.M, Levy. E, Marsan. S, et al. Multicenter study of a frozen glove to prevent docetaxel-induced onycholysis and cutaneous toxicity of the hand. J. Clin. Oncol 2005; 23:4424-9.

[9] Lehoczky 0, PulayT. Transverseleukonychia secondarytopaclitaxel-carboplatin chemotherapy in a patient with ovarian cancer. J ObstetGynaecol 2002; 22:694.

Citation: Dr. Sundeep Chowdhry, Dr. Archana J Lokhande, Dr. Paschal D'Souza. Transient Remoulding of Nails as A Sequitur to Docetaxel. Archives of Dermatology and Skin Care. 2018; 1(1): 1-3.

Copyright: (C) 2018 Dr. Sundeep Chowdhry, Dr. Archana J Lokhande, Dr. Paschal D'Souza. This is an open access article distributed under the Creative Commons Attribution License, which permits unrestricted use, distribution, and reproduction in any medium, provided the original work is properly cited. 